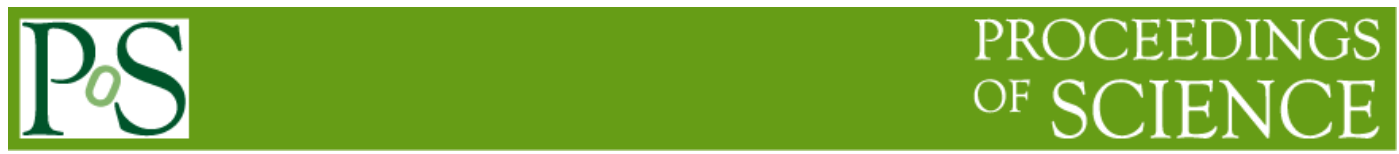

\title{
Self-Consistent Quantal Cranking Model for Monopole Excitations in Light Nuclei
}

\author{
P. Gulshani ${ }^{1}$ \\ NUTECH Services \\ 3313 Fenwick Cres., Mississauga, Ontario, Canada L5L 5N1 \\ matlap@bell.net
}

\begin{abstract}
We model collective monopole excitations (i.e., $0^{+}$excited states) in nuclei as oscillations in the nuclear radius. Assuming a monopole-intrinsic product wavefunction and transforming the many-particle shell-model Schrodinger equation accordingly, we obtained a Schrodinger equation that is a sum of monopole and intrinsic parts and a cross term that couples these parts. To fully account for the coupling between the monopole and intrinsic motions, we have applied a constrained variational method to the monopole-intrinsic Schrodinger equation to resolve it into two coupled time-reversal invariant crankingtype Schrodinger equations, one for the monopole oscillations and another for the intrinsic motion. For a harmonic oscillator mean-field potential, the equations are solved for the cranked parameters including the energy. The monopole frequency is determined from an energy-weight sum rule and the cranked equations. The impact of the monopole constraint on the intrinsic system is also investigated approximately. The excitation energy of the first excited $0^{+}$state is calculated and compared with that observed experimentally in the light nuclei.
\end{abstract}

$X$ Latin American Symposium on Nuclear Physics and Applications (X LASNPA)

December 1-6 2013, Montevideo, Uruguay

${ }^{1}$ Speaker 


\section{Introduction}

Light nuclei exhibit first excited $0^{+}$states with an excitation energy between 1 and 20 $\mathrm{MeV}$, which decreases with the mass number $A$. There has been a number of theoretical studies of these states for some of the light nuclei using various methods (refer to [1,2,3] for the relevant literature). However, the physical nature of these excitations are not yet fully understood at least in some of the nuclei. Previously [1], we modeled the monopole excitations in nuclei as oscillations in the nuclear radius in two dimensional space by transforming the many-particle Schrodinger equation to the nuclear radius co-ordinate. We determined the excitation energy using the underlying dynamical $s u(1,1)$ algebra. In this article, we generalize the previous study to three dimensions and use a variational method to derive two coupled selfconsistent cranking-type Schrodinger equations, one of the monopole and another for intrinsics motions. The monopole oscillation frequency is determined from these equations and an energy-weighted sum rule.

\section{Derviation of coupled monopole and intrinsic Schrodiner equations}

We use a nuclear product wavefuction according to the ansatz:

$$
\Phi=F(R) \cdot \phi\left(x_{n i}\right)
$$

where $F$ is the monopole oscillation wavefunction of the nuclear radius $R \equiv \sum_{n=1}^{A} r_{n}^{2} \equiv \sum_{n=1}^{A}\left(x_{n}^{2}+y_{n}^{2}+z_{n}^{2}\right)$, and $\phi$ is a rotationally invariant intrinsic wavefunction of the particle coordinates $x_{n i}(n=1,2, \ldots \mathrm{A}, \mathrm{i}=1,2,3)$ and subject to the constraint: $\frac{\partial}{\partial R} \phi=0$ (refer to [2] for more detail). Transforming the many-particle Schrodinger equation according to Eq. (1), we obtain:

$$
\phi \cdot\left(-4 R \frac{d^{2}}{d R^{2}}+b_{m}^{2} R\right) \cdot F-4 \frac{d F}{d R} \cdot \tilde{B} \phi+F \cdot \sum_{n=1}^{A}\left(-\nabla_{n}^{2}+b_{s}^{2} r_{n}^{2}\right) \cdot \phi=\varepsilon F \phi
$$

where $\tilde{B} \equiv \frac{1}{2} \sum_{n, j=1}^{A, 3}\left(x_{n j} \frac{\partial}{\partial x_{n j}}+\frac{\partial}{\partial x_{n j}} x_{n j}\right)$ and $\varepsilon$ is the reduced energy, defined on the right-hand side of Eq. (2). In Eq. (2), we have replaced the particle-particle interaction by its Hatree-Fock mean-field potential, commonly chosen to be the shell-model harmonic oscillator potential $\hat{V}_{\text {os }}$, which we have partitioned into the following form:

$$
\frac{2 M}{\hbar^{2}} \hat{V}_{o s} \equiv b^{2} \cdot \sum_{m=1}^{A} r_{m}^{2} \equiv b_{m}^{2} \cdot R+b_{s}^{2} \cdot \sum_{m=1}^{A} r_{m}^{2}
$$

where $b \equiv \frac{M \omega}{\hbar}$ and $\hbar \omega=41 A^{-1 / 3}$. In this article, we set $b_{s}=b$. The monopole frequency $b_{m}$ is determined in Section 5. 


\section{Derivation of self-consistent cranking model for coupled monopole-intrinsic motion}

We now take the expectation of Eq. (2) with respect to $F$ and $\phi$ and apply the RayleighRitz variational method to the resulting equation subject the wavefunction normalization and energy minimization $\partial \varepsilon / \partial F^{*}=\partial \varepsilon / \partial \phi^{*}=0$ to obtain:

$$
\begin{aligned}
& \left(-4 R \frac{d^{2}}{d R^{2}}+\beta_{R} \frac{d}{d R}+b_{m}^{2} R\right)|F\rangle=\left(\varepsilon-t_{o s}-b_{s}^{2}\left\langle\phi\left|\sum_{n=1}^{A} r_{n}^{2}\right| \phi\right\rangle\right)|F\rangle \\
& \left(-\sum_{n=1}^{A} \nabla_{n}^{2}+\beta_{o s} \cdot \tilde{B}+b_{s}^{2} \sum_{n=1}^{A} r_{n}^{2}\right)|\phi\rangle=\left(\varepsilon-t_{R}-b_{m}^{2}\langle F|R| F\rangle\right)|\phi\rangle \\
& a \cdot \beta_{o s}=\varepsilon-t_{o s}-t_{R}-b_{m}^{2}\langle F|R| F\rangle-b_{s}^{2}\left\langle\phi\left|\sum_{n=1}^{A} r_{n}^{2}\right| \phi\right\rangle
\end{aligned}
$$

where $\quad a \equiv\langle\phi|\tilde{B}| \phi\rangle, \beta_{o s} \equiv-4\left\langle F\left|\frac{d}{d R}\right| F\right\rangle, \quad t_{o s} \equiv\left\langle\phi\left|\left(-\sum_{n=1}^{A} \nabla_{n}^{2}\right)\right| \phi\right\rangle, \quad$ and $\quad t_{R} \equiv\left\langle F\left|\left(-4 R \frac{d^{2}}{d R^{2}}\right)\right| F\right\rangle$.

Eq. (6) is obtained from the expectation of either Eq. (4) or (5). Simultaneous solution of Eqs. (4), (5), and (6) determines the three parameters $\varepsilon, a$, and $\beta_{o s}$. Eqs. (4) and (5) may be viewed as a microscopic, self-consistent, cranking Schrödinger equations for the monopole and intrinsic motions respectively, with the cranking parameters $a$ and $\beta_{\text {os }}$ being dynamical variables determined self-consistently by the two motions. Another consequence of the selfconsistency is that Eqs. (4) and (5) are time-reversal invariant because $a$ and $\beta_{o s}$ are dynamical variables, which must be chosen to have real values because the operators $d / d R$ and $\tilde{B}$ associated with $a$ and $\beta_{o s}$ are real and non-hermitian. These features require us to choose real (and not unitary) solutions of Eqs. (4) and (5). These features are improvements over the conventional phenomenological cranking models $[4,5,6]$ where the cranking parameters are constant numbers and hence the models violate time-reversal invariance. Eq. (4) is a microscopic, self-consistent, and arbitrary-amplitude quantum generalization of the phenomenological, semi-classical Bohr Hamiltonian for the vibrational motion of a spherical nucleus [7]. This connection becomes more transparent when $R$ is identified with the Bohr vibrational parameter $\beta^{2}$. (Refer to [2] for a more detail discussion of these and other features of the model.) 


\section{Self-consistent solutions of Eqs. (4), (5), and (6)}

Real eigenfuctions and eigenvalues of the differential Eqs. (4) and (5) are obtained from the literature. These eigenfunctions are related to respectively confluent hypergeometric or Kummer and harmonic oscillator functions, with the restriction that $a>1$ to ensure vanishing of certain quantities on the system boundaries (refer to [2] for a more detail). The corresponding eigenvlaues are determined to be:

$$
\varepsilon-t_{o s}-b_{s}^{2}\left\langle\phi\left|\sum_{n=1}^{A} r_{n}^{2}\right| \phi\right\rangle=2 b_{m} \cdot(2 n+a), \quad \varepsilon-t_{R}-b_{m}^{2}\langle F|R| F\rangle=2 \Sigma \sqrt{b_{s}^{2}+\beta_{o s}^{2} / 4}
$$

where $\Sigma \equiv \sum_{n, k=1}^{n_{f}, 3}\left(n_{k}+\frac{1}{2}\right)$ is the total oscillaltor particle occupation number and $n_{f}$ is the Fermi level oscillator quantum number. Using these eigenfunctions and their generating functions, we determine the quantities:

$$
t_{o s}=\frac{b_{s}^{2} \cdot \Sigma}{\sqrt{b_{s}^{2}+\beta_{o s}^{2} / 4}}, t_{R}=b_{m} \cdot\left(2 n+a-B_{n}\right),\langle F|R| F\rangle=\frac{2 n+a}{b_{m}} \text {, and }\left\langle\phi\left|\sum_{n=1}^{A} r_{n}^{2}\right| \phi\right\rangle
$$

where $B_{n} \equiv \frac{2 \cdot n !}{(a+1)(a+2) \ldots .(a+n-1)} \sum_{k=0}^{n} \frac{(a-1) a \ldots .(a+n-k-2)}{(n-k) !}$.

Combining Eqs. (6), (7), and (8), we determine the following expressions. The total energy of the combined system is:

$$
\varepsilon=2 \Sigma \sqrt{b_{s}^{2}+\beta_{o s}^{2} / 4}+2 b_{m} \cdot\left(2 n+a-\frac{B_{n}}{2}\right)
$$

For $n=0$ with $B_{\mathrm{n}}=2$ :

$$
\beta_{o s}^{2}=\frac{2 b_{m}^{2}}{\Sigma^{2}}\left(1+\sqrt{1+\frac{4 b_{s}^{2} \Sigma^{2}}{b_{m}^{2}}}\right), \quad a=2 \Sigma\left(1+\sqrt{1+\frac{4 b_{s}^{2} \Sigma^{2}}{b_{m}^{2}}}\right)^{-1 / 2}
$$

For $n=1$ with $B_{\mathrm{n}}=2 \mathrm{a}$ :

$$
\beta_{o s}=2 b_{m}, \quad a=\frac{b_{m} \cdot \Sigma}{b}
$$

From Eq. (9), the excitation energy of the first excited $0^{+}$state is naturally defined by:

$$
\Delta E_{m} \equiv \frac{\hbar^{2}}{2 M}[\varepsilon(n=1)-\varepsilon(n=0)]=\frac{\hbar^{2} b}{2 M} \cdot \frac{\varepsilon(n=1)-\varepsilon(n=0)}{b}=\frac{\hbar \omega}{2} \cdot \frac{\varepsilon(n=1)-\varepsilon(n=0)}{b}
$$




\section{Determination of monopole frequency $b_{m}$ using sum rule and constrained intrinsic system}

To obtain a prescription for the monopole frequency $b_{m}$, we substitute in succession the eigenfunctions $F$ and $\phi$ for the eigenstates $|v\rangle$, the Hamiltonians in Eqs. (4) and (5) for $H$, and $R$ for the operator $\hat{A}$ in the energy-weighted sum-rule formula:

$$
\sum_{v=0}\left(E_{v}-E_{o}\right)|\langle v|\hat{A}| 0\rangle|^{2}=\sum_{v=0}\left(E_{v}-E_{o}\right)\langle 0|\hat{A}| v\rangle\langle v|\hat{A}| 0\rangle=\frac{1}{2}\langle 0|[[\hat{A}, H], \hat{A}]| 0\rangle
$$

Thereby we thereby obtain the formula:

$$
\frac{\Sigma}{\sqrt{b_{s}^{2}+\beta_{o s}^{2}(n=0) / 4}}=\frac{a(n=0)}{b_{m}}
$$

for $b_{m}$. Substituting Eq. (10) into Eq. (14), we obtain $\tilde{b}_{m}=\frac{\Sigma}{\Sigma^{2}-1}$. This formula yields unrealistically low values for $b_{m}$. To obtain a more realistic value of $b_{m}$, we have approximately imposed the constraint $\frac{\partial}{\partial R} \phi=0$ on the intrinsic wavefunction $\phi$ in the form of the expectation (i.e., the first moment) of the constraint and included it in the formulation in Sections 2 to 3 using the method of Lagrange multiplier and first-order perturbation theory. For ${ }_{2}^{4} \mathrm{He}$, we have cast the results of this analysis into the following convenient form for the quantities in Eqs. (7) and (8):

$$
\varepsilon-t_{R}-b_{m}^{2}\langle F|R| F\rangle=2 \Sigma^{1.08} \sqrt{b_{s}^{2}+\beta_{o s}^{2} / 4}, t_{o s}=\frac{b_{s}^{2} \cdot \Sigma^{1.17}}{\sqrt{b_{s}^{2}+\beta_{o s}^{2} / 4}},\left\langle\phi\left|\sum_{n=1}^{A} r_{n}^{2}\right| \phi\right\rangle=\frac{\Sigma^{0.8}}{\sqrt{b_{s}^{2}+\beta_{o s}^{2} / 4}}
$$

Using Eq. (15) and the steps given in Section 4, we obtain:

$$
\begin{array}{ll}
\beta_{o s}^{2}(n=0)=\frac{2 b_{m}^{2}}{\bar{\Sigma}^{2}}\left(1+\sqrt{1+\frac{4 b_{s}^{2} \bar{\Sigma}^{2}}{b_{m}^{2}}}\right) & a(n=0)=\sqrt{2} \bar{\Sigma}\left(1+\sqrt{1+\frac{4 b_{s}^{2} \bar{\Sigma}^{2}}{b_{m}^{2}}}\right)^{-1 / 2} \\
\beta_{o s}(n=1)=2 b_{m} & a(n=1)=\frac{b_{m} \bar{\Sigma}}{\sqrt{b_{s}^{2}+b_{m}^{2}}}
\end{array}
$$

where $\bar{\Sigma} \equiv \Sigma^{1.08}$. From Eqs. (14) and (16), we obtain

$$
b_{m}=b \Sigma^{-0.52}
$$

In this article, we use Eq. (17) also for the other light nuclei because the predictions in this article are of exploratory nature and more rigorous study will be given elsewhere. 


\section{Preliminary assessment of model for light nuclei}

Although the main objective of the analysis in this article is the derivation of the microscopic, quantal, self-consistent cranking model given in Sections (2) to (5), we have performed a preliminary scoping calculation of the excitation energy of the first excited $0^{+}$state in the light nuclei in the range $A \leq 120$. Using Eqs. (15), (16), and (17) and the values of $\hbar \omega=41 A^{-1 / 3}$ and $\Sigma \equiv \sum_{n, k=1}^{n_{f}, 3}\left(n_{k}+\frac{1}{2}\right)$, we have computed the excitation eneryg $\Delta E_{m}$ in Eq. (12). The results are presented in Table 1. Table 1 shows that, except for ${ }_{2}^{4} \mathrm{He}$ and ${ }_{6}^{12} \mathrm{C}$, the model significantly overpredicts the excitation energy because these nuclei are deformed in their first excited $0^{+}$states and, hence, each of these states forms either a member of quadrupole vibrational triplet or the band head of rotational band. The model ignores nuclear deformation. The good agreement for ${ }_{2}^{4} \mathrm{He}$ and ${ }_{6}^{12} \mathrm{C}$ may be accidental because of the approximations used to include the intrinsic constraint and the absence of the residual interaction.

\section{Concluding remarks}

We have modeled monopole excitations, manifested in the excited $0^{+}$states, in light nuclei as oscillations in the nuclear radius. By transforming the many-particle Schrodinger equation accordingly and using a monopole-intrinsic product nuclear wavefunction and a variational principle, we have derived coupled, self-consistent monopole-intrinsic, cranking-type, Schrodinger equations, which are time-reversal invariant, unlike the conventional phenomenological Inglis-type cranking equations. For a harmonic mean-field potential, these equations are solved for the cranking parameters including the energy eigenvalues. The monopole frequency is determined using an energy-weighted sum rule and an approximate imposition of the first moment of the monopole constraint on the intrinsic system. Preliminary application of the model shows that, except for ${ }_{2}^{4} \mathrm{He}$ and ${ }_{6}^{12} \mathrm{C}$, it significantly overpredicts the excitation energy of the first excited $0^{+}$states due to the neglect of nuclear deformation. Work is in progress to improve the prediction for spherical and deformed nuclei by: implementing the intrinsic constraint more accurately and including the residual two-body interaction, more realistic shell model mean-field potential such as Nilsson's and pairing interaction, center of mass motion, and nuclear deformation. The residual interaction introduces particle-hole excitations components in the intrinsic wavefunction. Indeed, it has been shown [3] using generator co-ordinate method that such 4 particle- 4 hole excitations are necessary to obtain good predictions for monopole excitation in ${ }_{8}^{16} \mathrm{O}$. 
Table 2. Predicted ( $\left.\Delta E_{m}\right)$ and Observed $\left(\Delta E_{\text {exp }}\right)$ first excited $0^{+}$state excitation energy in light nuclei

\begin{tabular}{|c|c|c|c|c|c|c|c|}
\hline Nucleus & $\begin{array}{c}\text { Ang. } \\
\text { mom./parity }\end{array}$ & $\begin{array}{l}\text { Multi- } \\
\text { polarity }\end{array}$ & $\tilde{b}_{m}$ & $\begin{array}{c}a \\
n=0 / n=1\end{array}$ & $\begin{array}{c}\tilde{\beta}_{o s} \\
n=0 / n=1\end{array}$ & $\begin{array}{c}\Delta E_{m}(\mathrm{MeV}) \\
\text { (\% overprediction) }\end{array}$ & $\begin{array}{l}\Delta E_{\exp } \\
(\mathrm{MeV})\end{array}$ \\
\hline${ }_{2}^{4} \mathrm{He}$ & $0^{+}$ & E0 & 0.3656 & $1.657 / 2.38$ & $0.47 / 0.73$ & $20.3(1 \%)$ & 20.1 \\
\hline${ }_{6}^{12} \mathrm{C}$ & $0^{+}$ & E0 & 0.1605 & $2.32 / 5.35$ & $0.14 / 0.32$ & $8.2(6 \%)$ & 7.7 \\
\hline${ }_{8}^{16} \mathrm{O}$ & $0^{+}$ & E0 & 0.1337 & $2.53 / 6.35$ & $0.11 / 0.27$ & $6.9(13 \%)$ & 6.1 \\
\hline${ }_{14}^{28} \mathrm{Si}$ & $0^{+}$ & E0 & 0.0866 & $3.09 / 9.53$ & $0.06 / 0.17$ & $4.9(-1 \%)$ & 4.97 \\
\hline${ }_{16}^{32} S$ & $0^{+}$ & E0 & 0.0789 & $3.23 / 10.39$ & $0.05 / 0.16$ & $4.6(21 \%)$ & 3.8 \\
\hline${ }_{20}^{40} \mathrm{Ca}$ & $0^{+}$ & E0 & 0.068 & $3.46 / 11.94$ & $0.04 / 0.14$ & $4.1(21 \%)$ & 3.4 \\
\hline${ }_{28}^{60} \mathrm{Ni}$ & $0^{+}$ & $E 0$ & 0.0496 & $4.0 / 15.97$ & $0.03 / 0.10$ & 3.4 (48\%) & 2.3 \\
\hline${ }_{28}^{62} \mathrm{Ni}$ & $0^{+}$ & E0 & 0.0485 & $4.04 / 16.32$ & $0.02 / 0.10$ & 3.3 (57\%) & 2.1 \\
\hline${ }_{32}^{70} \mathrm{Ge}$ & $0^{+}$ & E0 & 0.0445 & $4.21 / 17.67$ & $0.02 / 0.09$ & 3.2 (167\%) & 1.2 \\
\hline${ }_{40}^{90} \mathrm{Zr}$ & $0^{+}$ & E0 & 0.037 & $4.59 / 20.98$ & $0.02 / 0.07$ & $2.8(56 \%)$ & 1.8 \\
\hline${ }_{48}^{114} C d$ & $0^{+}$ & $E 0 / E 2$ & 0.0308 & $4.98 / 24.82$ & $0.01 / 0.06$ & 2.6 (136\%) & 1.1 \\
\hline${ }_{50}^{116} S n$ & $0^{+}$ & $E 0 / E 2$ & 0.0304 & $5.01 / 25.11$ & $0.01 / 0.06$ & $2.6(44 \%)$ & 1.8 \\
\hline${ }_{52}^{120} \mathrm{Te}$ & $0^{+}$ & $\begin{array}{l}\text { decay to final } \\
\text { states } 0^{+} / 2^{+}\end{array}$ & 0.0297 & $5.07 / 25.68$ & $0.01 / 0.06$ & 2.5 (127\%) & 1.1 \\
\hline
\end{tabular}

\section{References}

[1] P. Gulshani, su(1,1) model of first excited monopole states in light nuclei, Nuclear Physics A866 (2011) 37

[2] P. Gulshani, A microscopic quantal self-consistent cranking model for oscillations in spherical nuclei, arXiv 1401.5328 [nucl-th], January 21, 2014

[3] M. Bender and P.-H. Heenen, Beyond mean-field description of the low-lying spectrun of ${ }^{16} \mathrm{O}$, Nuclear Physics A713 (2003) 390

[4] D.R. Inglis, Phys. Rev. 96 (1954) 1059

[5] R. Rockmore, Response to dilatation and some soluble "squashing" models of interacting manyfermion systems, Phys. Rev. C12 (1975) 676

[6] D.J. Rowe, Nulcear Collective Motion, Mathuen, London 1970

[7] A. Bohr, Kgl. Dan. Mat. Fys. Medd. 26 (1952) 14 